\title{
A non-Abelian Chern-Simons term for non-BPS D-branes
}

\author{
Bert Janssen and Patrick Meessen \\ Instituto de Física Teórica \\ Universidad Autónoma de Madrid \\ C-XVI, C.U. Cantoblanco \\ 28049 Madrid, Spain \\ E-mail: 'bert. janssen@uam.es', patrick.meessen@uam.es'
}

ABstract: We propose a Chern-Simons term for $N$ coinciding non-BPS D-branes. Demanding full $U(N)$ invariance and compatibility with T-duality, it is shown that it is necessary to introduce new interaction terms, through which the non-BPS D-branes couple to all $p$-form RR fields.

KEywords: 'D̄-branes, T-duality'

\section{Introduction}

Non-BPS branes have recently attracted a lot of attention, since they might give us insight in the behaviour of $p$-branes without the protection of supersymmetry. Non-BPS branes have (open) tachyonic modes living on their world volume, due to the lack of supersymmetry, which make them in general unstable objects. However, when the tachyon potential has minima, very interesting phenomena can occur. It has been conjectured by Sen [1, [1] that at the stationary point of the potential, the negative energy of the potential and the posivite tension of the brane cancel each other, creating a state which is indistinguishable from the vacuum. Even more interesting things happen when the tachyon field interpolates between two minima of the potential: then, after condensation of the tachyon living on a (unstable) non-BPS-brane into a kink solution, the nonBPS brane reduces to a (stable) BPS $(p-1)$ -

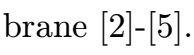

The dynamics of these non-BPS branes is given by their Born-Infeld (BI) and Chern-Simons (CS) action, including a tachyon field coupling, which distinguishes it from the actions of ordinary BPS branes. These actions have been

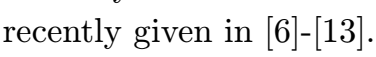

Another interesting topic that lately has be- come popular, is the phenomenon of gauge symmetry enhancement if we let $N$ (BPS) D-branes coincide [14]. As the $N$ D-branes approach each other, the open strings stretched between them become massless, the $N U(1)$ BI vectors are promoted to a $U(N)$ valued gauge field $A^{a}$ and the $(9-p)$ scalars $\Phi^{i}$ that describe the position of the branes in the transverse directions become scalars in the adjoint representations of $U(N)$, generating a non-commutative geometry in the transverse space.

The (non-commutative) BI and CS term for coinciding D-branes was recently given by $1 \overline{1}$. There it was argued by means of T-duality that some new terms should appear due to the nonAbelian character of the scalars $\Phi^{i}$. These new terms give rise to a non-trivial potential for the scalars, causing a kind of dielectric effect on the $\mathrm{D} p$-branes in the presence of higher form-fields [1 15 -

The aim of this letter is to unify the results of

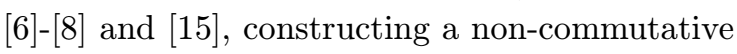

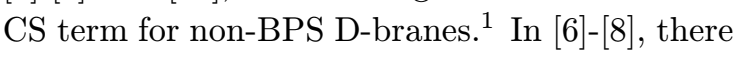
already have been given different proposals for CS terms for $N$ coinciding D-branes. However, since [1] ${ }_{1}^{1}$ - it has become clear that extra couplings and extra terms have to be taken into account to guarantee the $U(N)$ invariance of the ac-

\footnotetext{
${ }^{1} \mathrm{~A}$ proposal for a non-commutative, BI term for nonBPS D-branes was given recently in [10].
} 
tion and the correct behaviour under T-duality. In this letter we will try to give a more complete action, which takes these features into account.

The organisation of this letter is as follows: in the next two sections we will briefly review the CS term for coinciding (BPS) D-branes and for non-BPS branes and point out the subtleties occurring when trying to unify these. In section 4, we will present our proposal for the CS term for $N$ coinciding non-BPS D-branes and show that it is compatible with T-duality.

For simplicity, throughout this letter we will work in flat space-time with the Kalb-Ramond field $B_{\mu \nu}$ set equal to zero (i.e. neglecting all effects coming from the off-diagonal parts of the metric and its mixing with the Kalb-Ramond form in the T-duality rules). Later on in this letter, we will propose in analogy with [15], what we believe is the full CS term in the presence of $B$.

\section{A non-commutative CS term for BPS branes}

It is well known that BPS D $p$-branes couple to the $(p+1)$-form RR fields $[1 \overline{1}, 1,1 \overline{1}$, through their CS term in the following way: ${ }^{2}$

$$
S=\int_{p+1} C_{n} \wedge e^{F} .
$$

Here $F$ is the field strength of the $U(1) \mathrm{BI}$ vector living in the world volume of the D-brane and with $C_{n}$ we denote the pull-back of the (formal sum over) $n$-form RR fields, e.g.

$$
\begin{aligned}
C_{a_{1} a_{2}}=C_{\mu_{1} \mu_{2}} & \partial_{a_{1}} X^{\mu_{1}} \partial_{a_{2}} X^{\mu_{2}} \\
=C_{a_{1} a_{2}} & -2 C_{i\left[a_{1}\right.} \partial_{\left.a_{2}\right]} \Phi^{i} \\
& +C_{i_{1} i_{2}} \partial_{a_{1}} \Phi^{i_{1}} \partial_{a_{2}} \Phi^{i_{2}}
\end{aligned}
$$

where we have split the space-time coordinates $X^{\mu}$ in world volume coordinates $\xi^{a}$ and transverse coordinates $\Phi^{i}$.

It was pointed out in [1] 1 ind that a remarkable gauge symmetry enhancement occurs when $N$ parallel branes approach each other: the ground states of the strings stretched between the various D-branes become massless, the $N$ Abelian BI

\footnotetext{
${ }^{2}$ Modulo terms involving the A-roof hat genus, which we will neglect everywhere in this letter.
}

vectors become the components of a non-Abelian $U(N)$ vector and the scalars $\Phi^{i}$, indicating the position of the branes in the transverse direction, rearrange into a set of non-Abelian scalars, transforming in the adjoint representation of $U(N)$. In other words, the $U(1)^{N}$ symmetry group of the $N$ D-branes is enhanced to a $U(N)$ gauge group.

Recently it was shown how the CS term (2.1) gets modified under this effect [1] $\left.{ }_{1}^{1}\right]$. Of course, one obvious change is the necessity to take the trace over the $U(N)$ indices. But there is more: first of all, it turns out that all background fields should be functions of the non-commutative scalars $\Phi^{i}$. Secondly since the pull-back with respect to the transverse coordinates $\Phi^{i}$ has become $U(N)$-valued, the partial derivatives of $\Phi^{i}$ in (2.2) have to be replaced by covariant derivatives:

$$
D \Phi^{i}=\partial \Phi^{i}+i g\left[A, \Phi^{i}\right],
$$

where $A$ is the $U(N)$ gauge field with its field strength tensor given by

$$
F=d A+\frac{i g}{2}[A, A] .
$$

And, finally, it turns out that T-duality requires some extra interaction terms, due to the nonAbelian character of the scalars $\Phi^{i}$.

The CS term (2.1) is compatible with Tduality, in the sense that a T-duality transformation maps the term of a $\mathrm{D} p$-brane into the term of a $\mathrm{D}(p \pm 1)$-brane $\left[1 \overline{1}^{\prime}, \overline{1}_{1}^{\prime}\right.$. This is easily shown by mapping the CS term of the $\mathrm{D} p$ and $\mathrm{D}(p-1)$-brane, via double and direct dimensional reduction respectively, onto the same term in nine dimensions. ${ }^{3}$ Double dimensional reduction of the $\mathrm{D} p$-brane term gives: ${ }^{4}$

$$
S=\int_{p+1} \hat{C}^{+} \wedge e^{\hat{F}}=\int_{p}\left(C^{-}+C^{+} d \chi\right) \wedge e^{F}
$$

where the upper index + denotes the even (odd) RR-forms and the - the odd (even) RR-forms.

\footnotetext{
${ }^{3}$ This technique is the same as the one used to show the T-duality between the RR fields in Type IIA and B supergravity $[20,21]$.

${ }^{4}$ Performing dimensional reduction, it is necesarry to distinguish between ten- and nine-dimensional fields. We will do this by indicating the ten-dimensional ones with a hat. However in the parts where there is no confusion, the hats will be omitted.
} 
The nine-dimensional scalar field $\chi$, from the tendimensional point of view, comes from the component of the BI vector in the direction over which we reduce: $\chi=\hat{A}_{p}$. The nine-dimensional term is exactly the one obtained after direct dimensional reduction of the $\mathrm{D}(p-1)$-brane term

$$
S=\int_{p} \hat{C}^{-} \wedge e^{F}=\int_{p}\left(C^{-}+C^{+} d \chi\right) \wedge e^{F}
$$

but now the scalar $\chi$ comes from the reduction over the transverse direction: $\chi=\hat{\Phi}^{p}$. Note that in $(2.6), F$ is a world volume field therefore remains invariant under direct reduction. Thus the $p$-th component of the BI vector $\hat{A}_{p}$ gets mapped into the extra transverse direction $\hat{\Phi}^{p}$ (or vice versa) and hence the $\hat{F}_{a p}$ component of the field strength into the pull-back $\partial_{a} \hat{\Phi}^{p}$.

However, after the symmetry enhancement, non-trivial commutators appear in the pull-backs and field strengths and the rules for (double) dimensional reduction become more involved. The double dimensional reduction of the $\mathrm{D} p$-brane term now gives (compare to $(2.5)):^{5}$

$$
\begin{aligned}
S & =\int_{p+1} \operatorname{Tr}\left\{\hat{C}^{+} \wedge e^{\hat{F}}\right\} \\
& =\int_{p} \operatorname{Tr}\left\{\left(C^{-}+C^{+} d \chi+i g\left[\chi, \Phi^{i}\right] C_{i}^{+}\right) \wedge e^{F}\right\},
\end{aligned}
$$

where the last term now comes from the reduction of the non-Abelian pull-back

$$
\hat{D}_{p} \hat{\Phi}^{i}=i g\left[\chi, \Phi^{i}\right] .
$$

Clearly, an extra term has to be added to the term $\left(\underline{2} . \underline{6}_{1}^{6}\right)$ in order to reduce to the same ninedimensional term (2.7). The term

$$
S=\int_{p} \operatorname{Tr}\left\{\left(\hat{C}^{-}+i g \mathrm{i}_{\hat{\Phi}} \hat{i}_{\hat{\Phi}} \hat{C}^{-}\right) \wedge e^{F}\right\}
$$

does reduce in the correct way, where

$$
\begin{aligned}
\left(\mathrm{i}_{\Phi} \mathrm{i}_{\Phi} C^{(n)}\right)_{a_{1} \ldots a_{n-2}} & \equiv \Phi^{j} \Phi^{i} C_{i j a_{1} \ldots a_{n-2}} \\
& =\frac{1}{2}\left[\Phi^{j}, \Phi^{i}\right] C_{i j a_{1} \ldots a_{n-2}} .
\end{aligned}
$$

Applying again T-duality on (2.9i) shows the need to include a new term of the form

$$
\left(i g \mathrm{i}_{\hat{\Phi}} \mathrm{i}_{\hat{\Phi}}\right)^{2} \hat{C}^{+}
$$

${ }_{1}^{5} \mathrm{By}$ Tr we mean the symmetric trace description of $[22,123,151$. and an iterative procedure gives the fully T-duality invariant CS term: ${ }^{6}$

$$
S=\int_{p+1} \operatorname{Tr}\left\{e^{i g \mathrm{i}_{\Phi} \dot{\mathrm{i}}_{\Phi}} C \wedge e^{F}\right\} .
$$

Thus we see that the $N$ coinciding $\mathrm{D} p$-branes not only couple to the $(p+1)$-form RR fields and the lower $(p-1, p-3, \ldots)$ RR-forms via the $F$ as in the Abelian case, but also to higher $(p+3$, $p+5, \ldots) \mathrm{RR}$-forms via the the extra contraction terms $(2 . \overline{1}-\overline{1})$. It is these contraction terms that give rise to multipole moments and lead to a kind of polarisation effect of D-branes in the present of the external RR field [1 $\left.15 \overline{5}_{1}\right]$.

\section{Non-BPS branes and their pro- posed CS term}

Unlike the well-known supersymmetric D-branes, a (single) non-BPS D $p$-brane couples to the $p$ form RR field [inj] and therefore even (odd) nonBPS D $p$-branes appear in Type IIB (IIA) string theory.

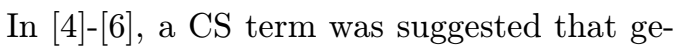
nerates this coupling:

$$
S=\int_{p+1} C_{n} \wedge d T \wedge e^{F}
$$

The form of this term was argued from string scattering amplitudes and the fact that after tachyon condensation, $(\underline{\mathbf{3}} . \overline{-1})$ ) reduces correctly to the well-known CS term for BPS D-branes [1] The authors of $[6]$ also give a generalisation to the non-Abelian case of $N$ coinciding D-branes, by taking the trace over the indices of the $U(N)$ symmetry group:

$$
S=\int_{p+1} C_{n} \wedge d \operatorname{Tr}\left\{T \wedge e^{F}\right\}
$$

where $T$ is in the adjoint representation of $U(N)$.

In [7] it was shown that for a system of $N$ coinciding D-branes and anti-D-branes, an interaction term with higher powers of the tachyon is needed in the CS term. This leads, before tachyon condensation, to the generalisation of

\footnotetext{
${ }^{6}$ The existence of these terms were also shown through matrix theory calculation in [24, 25$]$.
} 


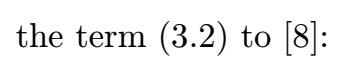

$$
\begin{aligned}
S & =\sum_{k, l} a_{k l} S_{k l}, \\
S_{k l} & =\int_{p+1} C_{n} \wedge \operatorname{Tr}\left\{(D T)^{2 k+1} T^{2 l} \wedge e^{F}\right\},
\end{aligned}
$$

where the coefficients $a_{k l}$ are undetermined numerical constants and

$$
D T=d T+i g[A, T] .
$$

It was shown that this term reproduces correctly the CS term for BPS D-branes after tachyon condensation.

Yet one can try to go a step further in the attempt to construct a non-Abelian CS term and demand invariance under T-duality. As shown in section $\overline{2}_{-v}^{2}$ the pull-back of the bulk fields have to be covariantized and extra interaction terms have to be included in order to have a correct behaviour under T-duality. In the next section we will try to construct such a term.

\section{A fully $U(N)$-invariant CS term for non-BPS D-branes}

Our proposal for the CS term of $N$ coinciding non-BPS $\mathrm{D} p$-branes is

$$
\begin{array}{r}
S=\sum_{k l} a_{k l} \int_{p+1} \operatorname{Tr}\left\{e^{i g \mathrm{i}_{\Phi} \mathrm{i}_{\Phi}} C\left[-i g \overleftarrow{1}_{[\Phi, T]}+D T\right] \times\right. \\
\left.\times(D T)^{2 k} T^{2 l} e^{F}\right\}
\end{array}
$$

where

$$
\left(C^{(n)} \overleftarrow{1}_{[\Phi, T]}\right)_{a_{1} \ldots a_{n-1}}=C_{a_{1} \ldots a_{n-1} i}\left[\Phi^{i}, T\right] .
$$

Like in section 2 rect behaviour under T-duality through double and single dimensional reduction. Let us first consider the case where $k=l=0$, which corresponds to a generalisation of the term (3.2):

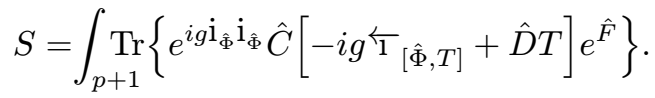

Double dimensional reduction of the second term gives

$$
\begin{aligned}
& e^{i g \mathrm{i}_{\Phi} \mathrm{i}_{\Phi}} \hat{C}^{+} \hat{D} T e^{\hat{F}}=\left\{-e^{i g \mathrm{i}_{\Phi} \dot{\mathrm{i}}_{\Phi}} C^{-} D T\right. \\
& \quad+i g e^{i g \mathrm{i}_{\Phi} \mathrm{i}_{\Phi}} C^{+}[\chi, T]-e^{i g \mathrm{i}_{\Phi} \mathrm{i}_{\Phi}} C^{+} D \chi D T \\
& \left.\quad+i g e^{i g \mathrm{i}_{\Phi} \dot{\mathrm{i}}_{\Phi}} C^{+} \overleftarrow{1}_{[\Phi, \chi]} D T\right\} e^{F},
\end{aligned}
$$

where again $\chi=\hat{A}_{p}$. The reduction of the first term gives

$$
\begin{aligned}
& e^{i g \mathrm{i}_{\Phi} \mathrm{i}_{\Phi}} \hat{C}^{+} \overleftarrow{\mathrm{I}}_{[\hat{\Phi}, T]} e^{\hat{F}}=\left\{-e^{i g \mathrm{i}_{\Phi} \mathrm{i}_{\Phi}} C^{-} \overleftarrow{\mathrm{I}}_{[\Phi, T]}\right. \\
& +e^{i g \mathrm{i}_{\Phi} \mathrm{i}_{\Phi}} C^{+} \overleftarrow{1}_{[\Phi, T]} D \chi \\
& \left.-i g e^{i g \mathrm{i}_{\Phi} \mathrm{i}_{\Phi}} C^{+} \overleftarrow{1}_{[\Phi, T]} \overleftarrow{1}_{[\Phi, \chi]}\right\} e^{F} .
\end{aligned}
$$

On the other hand, the single reduction of the $\mathrm{D}(p-1)$-brane CS term yields the terms

$$
\begin{aligned}
e^{i g \mathrm{i}_{\Phi} \mathrm{i}_{\hat{\Phi}}} \hat{C}^{-}= & e^{i g \mathrm{i}_{\Phi} \dot{\mathrm{i}}_{\Phi}} C^{-}+e^{i g \mathrm{i}_{\Phi} \mathrm{i}_{\Phi}} C^{+} D \chi \\
& -i g e^{i g \mathrm{i}_{\Phi} \mathrm{i}_{\Phi}} C^{+} \overleftarrow{\mathrm{I}}_{[\Phi, \chi]},
\end{aligned}
$$

$$
\begin{aligned}
e^{i g \mathrm{i}_{\tilde{\Phi}} \mathrm{i}_{\hat{\Phi}}} \hat{C}^{-\overleftarrow{1}_{[\hat{\Phi}, T]}}= & e^{i g \mathrm{i}_{\Phi} \mathrm{i}_{\Phi}} C^{-\overleftarrow{1}_{[\Phi, T]}} \\
& -e^{i g \mathrm{i}_{\Phi} \mathrm{i}_{\Phi}} C^{+} \overleftarrow{1}_{[\Phi, T]} D \chi \\
& +i g e^{i g \mathrm{i}_{\Phi} \mathrm{i}_{\Phi}} C^{+} \overleftarrow{1}_{[\Phi, T]} \overleftarrow{1}_{[\Phi, \chi]} \\
& -i g e^{i g \mathrm{i}_{\Phi} \dot{\mathrm{i}}_{\Phi}} C^{+}[\chi, T]
\end{aligned}
$$

Given that the world volume fields $D T$ and $F$ do not change under single reduction, we see that the terms (4.4)-(4.5i) obtained from the double reduction of the $\mathrm{D} p$-brane CS, coincide with the terms (4.6i)-(4.7) coming from the single reduction of the $\mathrm{D}(p-1)$-brane. This proves the Tduality invariance of (4.3i).

Comparing (4.4) and (4.7), it is clear why the extra term

$$
e^{i g \mathrm{i}_{\hat{\Phi}} \mathrm{i}_{\hat{\Phi}} \hat{C}^{+} \overleftarrow{1}_{[\hat{\Phi}, T]}}
$$

has been introduced: double reduction of the second term in (4.3i) yields a term proportional to the commutator $[\chi, T]$, as a result of the reduction of $\hat{D} T$ (analogous to $(2.8 \mathrm{j})$ ) and this term can only be compensated by a term of the form (4.7).

The generalisation of the proof to $k, l \neq 0$ is straightforward: the only extra contributions come from double reduction the $(\hat{D} T)^{2 k}$ terms. It is easy to see that

$$
\begin{aligned}
(\hat{D} T)^{n} & =\left(D T+i g[\chi, T] d \xi^{p}\right)^{n} \\
& =(D T)^{n-1}\left(D T+i g[\chi, T] \delta_{n, 2 k+1} d \xi^{p}\right),
\end{aligned}
$$

where the commutator term is only different from zero for odd power of $\hat{D} T$, due to the anti-symmetric character of the wedge product and the 
symmetric trace prescription. Hence the con' tribution of $(4.91)$ factorizes_into a term already present in the $k, l=0$ case and an extra overall $(D T)^{2 k}$. This completes the prove for the Tduality invariance of our proposed CS term (14.1).

Finally we will extend the CS term (4.11) in the presence of the Kalb-Ramond field $B$. In : analogy with [1]

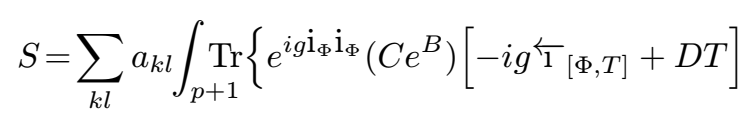

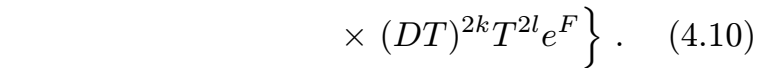

It would be interesting to see whether the proposed action, in combination with the BI term of [1] 10], contains solutions with dynamical tachyon condensation and a kind of polarisation effect on the non-BPS branes. We leave this for further investigation.
[9] N.D. Lambert, I. Sachs, IJ. High Energy Phys.। $\mathbf{0 3}(2000) 28$, hep-th/0002061!

[10] M. R. Garousi, Tachyon couplings on nonBPS D-branes and Dirac-Born-Infeld action, hep-th/0003122.

[11] E. Bergshoeff, M. de_Roo,_T. C. de Wit,_E. Eyras, S. Panda, 1]. High Energy Phys. 05! (2000) 009, 'hep-th/0003221'

[12] J. Klusoň, Proposal for non-BPS D-brane action, 'hep-th/0004106!

[13] N.D. Lambert, I. Sachs, IJ. High Energy Phys.1 $08(2000) 024^{\prime}$, hep-th/0006122,

[14] E. Witten, iNucl. Phys. B $\mathbf{4 6 0}(1996)$ 335, hep-th/9510135.

[15] R. Myers, 'J. High Energy Phys. 12 (1999) 022, hep-th/9910053.

[16] J. Polchinski, iPhys. Rev. Lett. 75 (1995) 4724, hep-th/9510017.

[17] M. Green, J. Harvey, G. Moore, 'Class. and Quant. Grav. 14 (1997) 477, hep-th/9605033.

[18] E. Alvarez, J.L.F. Barbón, J. Borlaf, iNucl.' Phys. B_479 (1996)_218, 'hep-th/9603089'.

The_authors_thank_C_Gómez, T_ _Ortín and_P. Silva for the usefull discussions. This work has ' been partially_supported_by the _TMR_ program

FMRX-CT96-0012 on Integrability, non-pertur'bative effects, and symmetry in quantum field theory. :- -

\section{References}

[1] A. Sen, 'J. High Energy Phys. 08 (1998) 010', hep-th/9805019.

[2] A. Sen, 'J. High Energy Phys. 08 (1998) 012', $\mathrm{I}^{-}-\mathrm{h}$ -

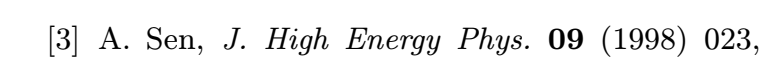

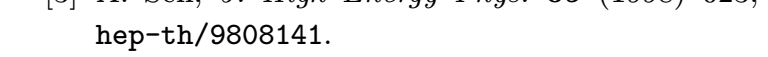

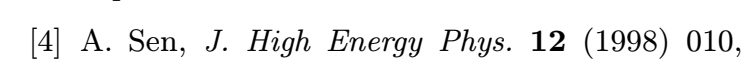
hep-th/9812031.

[5] P. Hořava, 'Adv. Theor. Math. Phys. 2 (1999), : - 1373, hep-th/9812135.

[6] M. Billó, B. Craps, F. Roose, 'J. High Energy Phys. 06 (1999) 033, hep-th/9905157.

[7] C. Kennedy, A. Wilkins, 'Phys. Lett. B 464' - (1999) 206, hep-th/9905195.

[8] J. Klusoň, D-Branes in Type IIA and Type IIB Theories from Tachyon Condensation, hep-th/0001123.
[19] E. Bergshoeff, M. de Roo, 'Phys. Lett. B 380' _1996) 265, hep-th/9603123'

[20] E. Bergshoeff, C. M. Hull, T. Ortín, iNucl. Phys.' B 451 (1995) 547, ihep-th/9504081

[21] P. Meessen, T. Ortín, iNucl. Phys. B 541 (1999)! 195 , inep-th/9806120!

[22] A.A. Tseytlin, iNucl. Phys. B 501 (1997) 4li, hep-th/9701125.

[23] A.A. Tseytlin, Born-Infeld action, supersymmetry and string theory, hep-th/9908105.

[24] W. Taylor, M. Van Raamsdonk, iNucl. Phys. $\mathbf{5 5 8}(1999) 63$, hep-th/9904095'.

[25] W. Taylor, M. Van Raamsdonk, iNucl. Phys. $\overline{\mathbf{B}}_{1}^{\prime}$ $573(2000) 703$, hep-th/9910052. 